\title{
CORRECCIÓN Y COGNITIVISMO. COMENTARIOS AL ARGUMENTO DE LA PRETENSIÓN DE CORRECCIÓN DE ROBERT ALEXY*
}

\author{
George Pavlakos ** \\ Universidad de Amberes-Universidad de Glasgow
}

RESUMEN. El argumento de la pretensión de corrección ha sido presentado por R. ALEXY con el objeto de defender la idea según la cual los enunciados normativos admiten respuestas objetivas. Mi propósito en este trabajo es el de preservar esta aspiración inicial, aun a costa de desviarme de algunas de las ideas originales que soportan el argumento. Empiezo explicando una versión pura del cognitivismo normativo, con base en la cual propongo reconstruir el argumento de la pretensión de corrección. Sostengo que el contexto de emisión de las emisiones normativas está dirigido a la posibilidad de cognición normativa, pero no la constituye. Lo que constituye tanto la posibilidad como la necesidad de cognición, es la estructura proposicional de las normas. Concluyo que el argumento de la pretensión de corrección debe salvaguardar una distinción entre el contexto de emisión de un enunciado normativo y la proposición que individualiza el contenido de la emisión.

Palabras clave: R. ALEXY, pretensión de corrección.

ABSTRACT. The argument from the claim to correctness has been put forward by R. ALEXY to defend the view that normative utterances admit of objective answers. My purpose in this paper is to preserve this initial aspiration even at the cost of diverting from some of the original ideas in support of the argument. I begin by spelling out a full-blooded version of normative cognitivism, against which I propose to reconstruct the argument from the claim to correctness. I argue that the context of uttering normative propositions points to the possibility of normative cognition, but does not constitute it. What constitutes the possibility of cognition is, as of necessity, the propositional structure of norms. I conclude that the argument from the claim to correctness ought to safeguard a distinction between the context of uttering a normative sentence and the proposition that individuates the content of the utterance.

Keywords: R. ALEXY, claim to correctness.

* Traducción de G. Villa Rosas, del original en inglés G. Pavlakos (2012), «Correctness and Cognitivism. Remarks on Robert Alexy's Argument from the Claim to Correctness», Ratio Juris, 25: 15-30.

** Profesor investigador, Universidad de Amberes y profesor, Universidad de Glasgow.

La elaboración de este ensayo fue generosamente financiada con recursos de la beca Odysseus (Odysseus Grant) de la Fundación para la Investigación del Estado de Flandes (Research Foundation Flanders - FWO). Algunas de las ideas presentadas en este escrito han sido desarrolladas a lo largo de años de conversaciones con R. Alexy, C. Heidemann y más recientemente con J. M. CABRA. Les doy las gracias por su valiosa contribución. También agradezco a CIRSFID y a Ratio Juris por haberme invitado a participar en la conferencia Law and Morality: the Perspective of Robert Alexy, que fue celebrada el 12 de noviembre de 2010 en Bolonia. Finalmente, quisiera agradecer los valiosos comentarios de la audiencia del Dokrorandenkolloquium del Departamento de Derecho Público y Filosofía del Derecho de la Universidad de Kiel, donde presenté el penúltimo borrador del documento en junio de 2011. 


\section{PRELIMINARES}

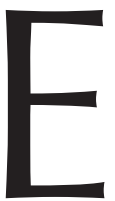

n una serie de ensayos de los años noventa K.-O. APEL sostuvo que él usaba a HABERMAS con el objeto de discurrir contra el mismo HABERMAS («mit Habermas gegen Habermas zu denken») (APEL, 1998, Chs. 11-13). A pesar de que posiblemente no puedo hacer una apuesta más alta que aquélla de APEL, yo quisiera también argüir de una forma similar acerca de ALEXY en contra de ALEXY. Sin embargo, con una diferencia importante: mientras que en sus ensayos APEL habitualmente utilizó la línea argumentativa de HABERMAS para negar su conclusión, yo seguiré el camino reverso: yo quiero defender las ideas fundamentales de ALEXY que subyacen al argumento acerca de la pretensión de corrección, y al mismo tiempo revisar, que no rechazar, el camino argumentativo hacia ella.

El argumento acerca de la pretensión de corrección (en adelante APC) ha adquirido el estatus de una justificación casi legendaria, dirigida a sostener que los enunciados normativos tienen una respuesta objetiva, en lugar de una respuesta subjetiva o una mera decisión o ninguna respuesta en absoluto. Cuando HABERMAS evoca de manera temprana esta justificación en relación con la explicación de la ética del discurso, dicha evocación tiene el propósito de argüir que los discursos prácticos tienen significado cognitivo, en el sentido de que son conducidos por premisas las cuales admiten valores de verdad. Mi propósito en este ensayo es preservar la aspiración inicial, la cual el APC está dirigido a defender, aún a costa de separarlo de algunas de las ideas que lo soportan. Al final creo haber aclarado y fortalecido el APC más que haberlo abandonado por completo - pero este veredicto final corresponderá al lector-.

Ésta será la estructura del ensayo: en la segunda parte adopto una versión pura del cognitivismo normativo y explico sus requerimientos. Yo sostengo que si la cuestión de la corrección es la de satisfacer la aspiración cognitivista, entonces dicha corrección debe estar de acuerdo con aquella versión paradigmática del cognitivismo. La tercera parte ofrece una interpretación y reconstrucción del APC de cara al cognitivismo en su versión pura. En este punto el APC es explicado como una estrategia dirigida a demostrar que el contexto de emisión de las proposiciones normativas está dirigido a la posibilidad de cognición normativa, pero no la constituye. Lo que constituye tanto la posibilidad como la necesidad de cognición, es la estructura proposicional de las normas (razones). El punto principal que aquí se plantea es que el APC debe salvaguardar la distinción entre el contexto de emisión de un enunciado normativo y la proposición que individualiza el contenido de la emisión. Finalmente la cuarta parte discute algunas consecuencias de la versión pura del cognitivismo para los actos de habla que afirman enunciados normativos.

\section{COGNITIVISMO PURO}

Yo sostengo que el éxito de todo programa cognitivista puede ser medido en relación a su capacidad para esclarecer nuestras razones normativas de una manera no psicologista. En resumidas cuentas, esta conclusión permite afirmar que sólo algunos tipos de cognitivismo tienen éxito, en tanto permiten explicar nuestras razones norma- 
tivas como poseedoras de poder motivacional de manera independiente de cualquier estado mental psicológico.

Se puede argumentar que el cognitivismo con respecto a la normatividad presupone algo como lo siguiente ${ }^{1}$ : lo que nos motiva a actuar, es decir, las razones que nos motivan (y por consiguiente el significado normativo de los conceptos que usamos en relación con aquellas razones), no se deriva de un estado mental (es decir, de entidades naturalistas), sino que es idéntico a nuestras razones normativas. Razones normativas son aquello de lo que tratan nuestros estados mentales, cuando aquéllos explican nuestro comportamiento (PARFIT, 2006, 365). En cierto sentido apropiado del término, nuestros estados mentales tratan de manera típica de hechos. De esto se sigue que: yo tengo una razón para $\mathrm{F}$ no en razón al hecho de que yo estoy en un estado mental particular, sino en razón al hecho específico del que trata mi estado mental.

Cambiando al nivel del discurso (de manera tal que permanezcamos dentro de los confines del giro lingüístico, el cual es mayormente preferido por las teorías éticas discursivas): las razones para la acción se integran en entidades susceptibles de valor de verdad, es decir, en proposiciones ${ }^{2}$, en oposición a actitudes proposicionales, es decir, creencias, deseos o estados volitivos en sentido amplio. Utilizaremos ejemplos concretos:

Razones motivantes como estados motivacionales:

(EM) La razón por la cual yo tomo una sombrilla es mi creencia en que va a llover.

De manera diversa, esto es lo que nosotros obtenemos cuando las razones motivantes son idénticas a los hechos:

(RM) La razón por la que yo tomo una sombrilla es (el hecho de) que llueve 3 .

Cambiando a un caso más complejo referido a un hecho normativo, el tipo de razón contrastante asume la siguiente forma:

(EM) La razón por la cual yo ayudo al necesitado es que yo creo/pretendo/deseo (que) ayudar al necesitado (porque) es bueno ${ }^{4}$.

(RM) La razón por la cual yo ayudo al necesitado es porque (el hecho de) ayudar al necesitado es bueno.

Se seguiría entonces que la «estructura» general de la razón motivante no-naturalista es:

El hecho «que F»

$\mathrm{NO}$

El hecho de mi creencia/deseo/ etc. «que F».

${ }^{1}$ Las formulaciones siguientes le deben mucho al reciente trabajo de PARFIT (PARFIT, 2006).

2 Además, si una proposición es verdadera, entonces un hecho existe.

3 Estrictamente hablando hay una premisa que falta aquí, que es la que pronuncia el valor de evitar mojarse. El hecho que informa la premisa que falta puede ser llamado, siguiendo a FitZPATRICK, 2009, una razón normativa autónoma mientras que el hecho de que llueve juega el rol de una condición que posibilita, la cual de manera concordante puede ser llamada razón posibilitante. Razones normativas autónomas son más complejas que las razones posibilitantes y, en última instancia, son las portadoras de la normatividad. Para una discusión detallada sobre razones normativas autónomas, vid. PAVLAKOS, 2011.

${ }^{4}$ Obviamente existe una asimetría entre creencias y deseos. El contenido de las creencias depende del valor de verdad de la proposición — por el contrario, en cuanto a los deseos, éste no es el caso-. Sin embargo, una creencia en sí misma no es una razón motivante como razón normativa; sólo la proposición verdadera (i.e. el hecho) que corresponde a ella lo es. 
Por consiguiente, en relación a las razones, un cognitivismo puro requiere que nuestras razones motivantes no sean los estados psicológicos que contienen una creencia/intención o un deseo, sino los hechos reales de los que tratan nuestras creencias, deseos, etc. Tales hechos constituyen nuestras razones normativas y, más allá, proveen el significado conspicuo o contenido de nuestras proposiciones de deber —el cual nosotros debemos cumplir-.

En resumen, debe ser elaborada una distinción entre estados motivacionales y razones motivantes: desde una perspectiva no-cognitivista (o mentalista), las razones motivantes se agotan en los estados motivacionales. Desde una perspectiva cognitivista las razones motivantes son independientes de cualquier estado mental (es decir, de actitudes proposicionales tales como creencias, deseos, etc.) e idénticas a los hechos que constituyen nuestras razones normativas. Ésto es lo que yo asumo como el punto fundamental del cognitivismo puro.

\section{LA PRETENSIÓN DE CORRECCIÓN Y LAS NORMAS COGNOSCENTES}

Afirmar las premisas de un cognitivismo normativo puro tiene cierto atractivo como ejercicio intelectual. Sin embargo, carece de toda fuerza apodíctica: aún si el cognitivismo es bueno, útil o conveniente, su valor resultará comprometido a menos que se muestre que él no es una entre muchas opciones, sino que por el contrario, que existen razones (no instrumentales) para optar por los hechos normativos como los hacedores de verdad de nuestras proposiciones normativas, como opuestos a todo estado mental naturalista.

Es en este punto en el que interviene el argumento de la pretensión de corrección - que ya se encuentra en el trabajo de HABERMAS, pero elaborado de manera prominente por R. AlEXY en el campo de la teoría general del derecho-. Su rol en relación a la fundamentación del programa cognitivista de la ética del discurso es precisamente el de asegurar y profundizar la idea de que los hechos normativos son la clase apropiada de entidades que hacen nuestras proposiciones normativas verdaderas, de manera antecedente a los estados mentales de quien pronuncia un enunciado normativo (HABERMAS, 2001, 57n.). En el caso de AlEXY, el APC fue introducido con la intención específica de demostrar una unidad necesaria entre el derecho y la moral, una tesis que es comúnmente conocida como la tesis de corrección:

[El APC] dice que las normas jurídicas individuales y las decisiones jurídicas individuales así como los sistemas legales como un todo presentan necesariamente una pretensión de corrección. Un sistema de normas que ni de manera explícita ni de manera implícita tenga esta pretensión no es un sistema jurídico. A este respecto, la pretensión de corrección tiene un significado clasificatorio. Los sistemas jurídicos que tengan de hecho esta pretensión pero fallen en satisfacerla son jurídicamente deficientes. A este respecto la pretensión de corrección tiene un significado cualificatorio. Un significado cualificatorio exclusivo está unido con la pretensión de corrección de normas jurídicas individuales y decisiones jurídicas individuales. Estas son jurídicamente defectuosas si no tienen la pretensión de corrección o si ellas fallan en satisfacerla (ALEXY, 2002a, 36).

Pero también en este caso, el éxito de esta tarea se basa en la premisa del cognitivismo normativo. Yo asumo que una no polémica interpretación del APC es aquella 
en virtud de la cual el derecho está necesariamente conectado con la moral en razón a que toda emisión de carácter normativo necesariamente posee un contenido cognitivo el cual es individualizado por hechos normativos idóneos ( $\mathrm{y}$, se debe añadir, que no hay nada inherente a aquellos hechos que sea peculiarmente jurídico) ${ }^{5}$. Además, ALEXY explícitamente concede algo entre líneas a la estrategia del cognitivismo puro, cuando él de manera general comenta sobre el estatus de las afirmaciones normativas (morales):

Enunciados morales que no planteen [la pretensión de corrección] no son juicios morales sino expresiones de emoción (ALEXY, 1999, 26).

Pero para que esto funcione hay que asegurarse que el APC pueda cumplir con el principal requisito del cognitivismo puro, cuya característica principal es la habilidad para mantener separadas las razones normativas de los estados motivacionales (como mentales).

Volvamos a la estructura del APC e indaguemos si a ella corresponde esta tarea. Yo ofreceré primero una reconstrucción del APC con el objeto de mostrar cómo él puede emplear el cognitivismo puro. Yo sostengo que él es la razón principal que permite el empleo del APC dentro del contexto más amplio de la teoría moral cognitivista, que es la ética del discurso. A continuación, examinaré algunas deficiencias del APC tal y como éstas emergen del debate entre sus críticos y ALEXY, deficiencias que amenazan con socavar la eficacia del argumento en tanto estrategia para sostener el cognitivismo. Mi objetivo será el de eliminar estas deficiencias y clarificar algunos otros malentendidos que pueden distorsionar el papel de la pretensión de corrección dentro del contexto más amplio de la teoría de la normatividad de ALEXY.

En primera instancia el APC apunta al hecho de que los enunciados normativos pueden ser afirmados. Si esto es verdad entonces se deduce de ello que tales enunciados tienen una estructura proposicional, y por consiguiente que ellos admiten una evaluación en términos de verdad y falsedad. Se deduce entonces que el hecho de la posibilidad de aseveración apunta a la necesidad de la estructura proposicional de los enunciados normativos. Que un enunciado normativo sea verdadero significa que, de hecho existe una obligación válida (o, de una forma más general, que él puede generar una genuina razón para la acción). Aunque las condiciones para crear una obligación pueden quedar por ahora abiertas, lo que es esencial en esta etapa es notar que existen razones de cierta clase que hacen a las proposiciones (normativas) verdaderas: esto es, existen hechos normativos, o normas ${ }^{6}$, los cuales deben mantenerse separados de las actitudes o pretensiones de otra especie ${ }^{7}$.

\footnotetext{
5 En esta medida, la carga de la prueba de la separación entre el derecho y la moral recaería en quien abogue por ello.

6 Tales hechos pueden ser llamados razones o normas. Para el caso de las razones, las cuales no pueden ser analizadas en este ensayo, yo prefiero el término «norma» sobre el término «razón», aunque ningún daño supone el uso de ellos de manera indistinta. «Norma» parece ser también la preferencia de ALEXY. El adopta lo que él mismo ha denominado concepción semántica de las normas. Según dicha concepción, las normas no se identifican con el elemento lingüístico particular (i.e. enunciados), que las expresa, sino con el significadocontenido objetivo (que es la proposición), que es expresado (AlEXY, 2002b, 21-25); vid. también PAVLAKOS, 2011.

${ }_{7}$ Los tres estadios del argumento se pueden presentar como las tres etapas de un argumento trascendental, con el fin de permanecer en línea con la intención de la ética del discurso y así otorgarle el dejo de una
} 
Lo que se sigue a partir de esta reconstrucción, es que existe un aspecto objetivo de la pretensión de corrección —llamado la dimensión proposicional ${ }^{8}$ - el cual no está a disposición de quien pronuncia el enunciado normativo pertinente. Para ser más preciso, ni la proposición, la cual individualiza el contenido del enunciado normativo, ni los motivos que hacen la proposición verdadera, son disponibles, en el sentido de ser dependientes de las condiciones de actuación del acto de lenguaje pertinente. Entonces si, dentro de otras cosas, el emisor no tiene la intención, o si falla en elevar la pretensión, sea ella implícita o explícita, o si eleva una pretensión diferente, o incluso si tiene la intención de contradecirla, la dimensión proposicional está destinada a permanecer inmune a todo esto 9 . Interpretar en esta perspectiva el APC puede perfectamente brindar los fundamentos necesarios para la posibilidad de los hechos normativos, como condición de un cognitivismo normativo puro, según lo expuesto anteriormente.

Es necesario advertir en este punto que al demostrar la necesidad de hechos normativos, en lugar de refutar los motivos de fondo, el APC refuta el mentalismo, o el nocognitivismo en la metaética. Él no descarta las actitudes mentales (creencias, deseos) por ser motivos de fondo defectuosos, sino que por el contrario, sostiene que dichas actitudes mentales son en general, una clase errónea de objetos cuando se trata de hacer cualquier proposición verdadera, en especial si ésta es normativa. En este caso lo que se requiere son hechos, ya que cuando una proposición es verdadera, entonces existe un hecho. Este punto es importante, por lo que me permitiré elaborarlo un poco más: concepciones mentalistas o no-cognitivistas de la normatividad (con preeminencia entre ellas la del expresivismo) se basan en una imagen semántica del lenguaje normativo, que asume que las proposiciones se derivan, en cuanto a su contenido, de las actitudes mentales de los agentes. Esta sui generis pretensión semántica es profundizada más o menos como sigue. En contraste con explicaciones semánticas estándar, el expresivismo identifica las condiciones semánticas de corrección no como condiciones de verdad sino como condiciones de aseverabilidad. Este modelo alternativo semántico —denominado por M. SCHROEDER como «expresivismo de aseverabilidad» por obvias razones (SCHROEDER, 2008, 28-35) - sostiene que el contenido de un enunciado se encuentra semánticamente asociado con la condición de que el emisor se encuentre en un estado mental ${ }^{10}$. Tomemos por ejemplo el enunciado S: «El dolor debe ser evitado». En una semántica veritativa, el significado de este enunciado sería individualizado por la proposición el dolor debe ser evitado, el cual de manera subsecuente determinaría el contenido del estado mental en el cual el emisor se encuentra, cuando expresa S. ¡Esto no ocurre en el expresivismo de aseverabilidad! Aquí el contenido de $\mathrm{S}$ es derivado

necesidad conceptual (HABERMAS, 2001; 2002a). En consecuencia, el hecho de que los enunciados normativos sean frecuentemente aserciones es una premisa fáctica débil. Luego, la segunda premisa afirma, que el hecho de que dichos enunciados posean una estructura proposicional es una condición para su funcionamiento como aserciones. Finalmente, se seguiría que los enunciados normativos necesariamente poseen una estructura proposicional.

${ }^{8}$ En estricto sentido la dimensión proposicional pertenece a los actos de habla más que a la pretensión. Con base en el argumento que aquí se presenta, esta dimensión es llevada a cabo debido a que se evoca la pretensión.

9 El único caso en el que el elemento performativo puede afectar el carácter proposicional de la emisión ocurre cuando la afirmación no alcanza a cumplir los criterios sintácticos y gramaticales de un enunciado bien formado (HeIDEMANN, 2005, 142).

${ }_{10}$ Dado el postulado de la consistencia, lo que corresponde al caso de los deseos, se aplica también al caso de las creencias. 
aún de la proposición el dolor debe ser evitado pero ésta no es la última instancia para adjudicar el contenido a S. Para que esta proposición sea en sí misma individualizada debe estar de acuerdo con el estado mental — creencia, deseo u otro- en el que se encuentra el emisor cuando expresa S, o citando a SCHROEDER: «En pocas palabras, podemos decir que el enunciado "expresa" éste [estado mental]» (SCHROEDER, 2008, 31). Por consiguiente, de acuerdo con esta perspectiva, los estados mentales son antecedentes a las proposiciones - y, por la misma razón, también a los enunciados- con respecto a su significado y contenido definitivos. Es precisamente este paso semántico el que requiere ser desmantelado antes de que el cognitivismo puro pueda ser asumido; y es el APC, al que corresponde ejecutar tal tarea de desmantelamiento.

Lamentablemente, el debate en torno al APC ha sido llevado a cabo en términos que confunden la dimensión proposicional de la pretensión de corrección con aquello que se puede llamar como su dimensión performativa. Por consiguiente, tanto el criticismo elevado en contra del APC como las defensas del mismo sostenidas por ALEXY, parecen pretermitir la tarea cognitivista clave del APC, esto es, su habilidad para bloquear toda fundamentación de la normatividad sobre objetos psicológicos inertes.

En particular, la mayoría de los críticos confunden la relación entre el acto de habla de la emisión de una proposición normativa y la proposición que es expresada. Es decir, ellos asumen la ejecución del acto de habla que afirma un enunciado normativo como constitutiva del contenido del enunciado que es afirmado. De esta manera, ellos identifican deficiencias que devienen posibles tan sólo si la dimensión performativa de la aserción es asumida como determinante de su dimensión proposicional. Por otra parte, los elementos de la ejecución, cuya presencia o carencia afectaría la ejecución de la aserción, son asumidos como si tuviesen impacto en la dimensión proposicional de la aserción. Dependiendo de la formación filosófica de cada crítico, este impacto es apreciado como positivo o rechazado como perjudicial.

Ésta es una muestra de los puntos de vista que se han presentado: ¿pueden las normas evocar una pretensión, o tan sólo las personas pueden hacerlo? y si sólo las personas pueden hacerlo, ¿no deviene entonces la pretensión demasiado subjetiva?, ¿qué sucede si las personas que toman parte en la práctica del derecho olvidan elevar la pretensión o incluso elevan una distinta? ${ }^{11}$. Además, si toda norma, como sostiene ALEXY, presenta necesariamente una pretensión de corrección, ¿cómo es posible que una norma continúe siendo legal aún cuando falle en erigir la pretensión de corrección (BulYGin, 1993, 21; id., 2000, 134)? En términos más generales, si la pretensión es necesariamente evocada en corrección con normas jurídicas (sea por las normas mismas o por los agentes que toman parte en la práctica) entonces no hay lugar a negar el carácter jurídico de una proposición jurídica. Finalmente, como otra crítica señala: si todo lo que es jurídico (sea un sistema o una norma) necesariamente implica la pretensión, entonces es perfectamente concebible un sistema jurídico que sea dirigido por un grupo de gente insensible, el cual no sólo yerre en elevar la pretensión sino que incluso

${ }^{11}$ C. Heidemann cita el caso de los contemporáneos de Galileo quienes, de manera irónica pronunciaron: «Si seguro, la tierra se mueve alrededor del sol», preguntándose por el impacto que puede tener, sobre la proposición que es afirmada, el hecho de que alguien se pronuncie de manera no seria, o incluso en broma (HEIDEMANN, 2005, 129). 
explícitamente la contradiga, o simplemente pronuncie «x está jurídicamente obligado, aun cuando dicha obligación sea inmoral» (HEIDEMANN, 2005, 142).

En todos estos casos, la crítica asume de una u otra forma que el contexto de ejecución se abre paso en el contenido de la proposición que es afirmada. Esto está sin embargo lejos de ser una suposición sólida. Si yo afirmo: «Se debe robar bancos», aun cuando la dimensión performativa de mi afirmación realice un esfuerzo por reducir la ambigüedad de «banco», haciendo que dicha afirmación se refiera a la institución, permanece siendo cierto que no existe permiso, y mucho menos que se debe empezar a robar bancos ${ }^{12}$.

Por el contrario, si la norma en cuestión no es otra que la proposición afirmada entonces, también es verdad, que los requerimientos para el éxito del acto de habla de aserción no pueden ser constitutivos del contenido de la norma (como proposición normativa verdadera). A lo sumo, ellos son un reflejo o un «síntoma» (HEIDEMANN, $2005,140)$ de que la norma tiene una estructura cognitiva. Más bien, en este caso es la norma la que es constitutiva de los requerimientos para la eficacia del acto de habla relevante ${ }^{13}$. La norma como proposición verdadera introduce un elemento cognitivo en el acto de habla, el cual es antecedente al contexto dentro del cual el enunciado relevante es afirmado (incluyendo la cuestión de si el emisor pretende evocar una u otra pretensión) ${ }^{14}$. C. HEIDEMANN lo ha puesto en términos sucintos:

$\mathrm{Si}$, sin embargo, la pretensión de corrección no es evocada [...] esto no tiene consecuencias necesarias para el significado-contenido normativo [GP: i.e. proposición] del enunciado o su validez (HEIDEMANN, 2005, 142).

En respuesta a los críticos, ALEXY introdujo en algún momento la distinción entre una evocación objetiva y una subjetiva de la pretensión, con el objeto de impedir el colapso del APC en la psicología del emisor (AlEXY, 1998, 206; id., 2007). En este contexto ALEXY sostiene que mientras la evocación subjetiva de la pretensión se refiere a personas reales, aquélla objetiva se refiere a agentes idealizados como participantes en el sistema jurídico. Por un lado, una persona evoca subjetivamente una pretensión si ella desea hacerlo. Por el lado contrario, una pretensión objetiva «no es un asunto privado, sino que está necesariamente relacionada con el papel de un participante en un sistema jurídico» (ALEXY, 1998, 206).

No obstante, de cara al cognitivismo en su versión pura, la distinción de ALEXY no alcanza a bloquear la contaminación con elementos performativos de la dimensión

12 Esta es una controversia clásica en filosofía, hasta qué punto las circunstancias contextuales dan forma al contenido de la proposición. Contextualistas parecen forzar esta influencia, mientras los no contextualistas, o literalistas se resisten a la estrategia contextualista (REVANATI, 2004; y para una aplicación en teoría jurídica: PAVLAKOS, 2009).

13 Vid. la siguiente parte sobre los requerimientos de la afirmación.

14 Se podría argumentar que la gran innovación de la teoría de los actos de habla fue precisamente su habilidad para ampliar el contenido semántico de las proposiciones a través de su enriquecimiento con elementos pragmáticos. Junto con esta función, los actos de habla resaltan, además de la comunicación, un elemento propio de la habilidad creativa incorporado en el pensamiento humano, en virtud del cual se pueden crear nuevas razones, ya sean ellas de creencia o de acción. No obstante, se debe ser cauteloso en otorgar a la mente humana una autoridad completa de contenido independiente en la creación de razones para la creencia y la acción. Una observación cautelosa hace evidente que tales razones deben ser poseedoras de un estatus mental independiente, si ellas deben valer como estándares fiables (PAVLAKOS, 2011). 
proposicional del APC. En contraposición, nosotros podemos calificar su propuesta como «cognitivismo débil». De acuerdo con dicha propuesta, el APC implica que sólo en la medida en que se pretende imparcialmente ${ }^{15}$ tener una particular postura respecto de un enunciado normativo, puede este último ser asumido como poseedor de un contenido proposicional objetivo. Lo que es, no obstante, decepcionantemente débil, porque ello presupone que el contenido de la norma es individualizado por (y en la medida permitida por) el estado mental en el cual se encuentra algún emisor. Una conclusión inmediata a partir de ello, es que el mismo enunciado acabaría significando diferentes proposiciones, cada vez que el contexto performativo variase. Para ilustrar el punto: si el emisor pretende bromear o no afirmar «que $p »$, entonces la afirmación no sería sobre $p$ sino sobre $p 1$. Con el fin de obtener $p$ a partir de «que $p$ »necesitaríamos evidenciar que el emisor pretendió afirmar «que $p{ }^{16}$.

El cognitivismo débil es deficiente cuando se trata de dar contenido a la distinción — crucial para el cognitivismo- entre estados mentales y los hechos que son nuestras razones normativas. La cuestión no es si las normas o las personas pretenden (aún necesariamente) expresar un enunciado con una intención asertórica (de hecho, yo estaría gustoso en admitir que en realidad sólo son personas quienes pueden pretender en este sentido). La cuestión es diferente: esto es, si, al inducir el modo asertórico, los enunciados normativos son necesariamente proposicionales (susceptibles de contenido cognitivo).

Es esta última necesidad, que conlleva el contenido proposicional, la que puede conferir la cantidad requerida de independencia mental del contenido normativo, que es obligatorio para el cognitivismo en sentido puro. Aquí la necesidad se adhiere al contenido cognitivo de los enunciados normativos, no a la existencia de cualquier característica actitudinal de aquél que afirma esos enunciados. De esto se sigue, si puedo afirmarlo con arrojo, que la posibilidad (no la realidad) de aserción es la que fundamenta la necesidad del cognitivismo. Entonces, cuando ALEXY sostiene: «Las normas individuales presentan necesariamente la pretensión de corrección (por consiguiente, siguen siendo jurídicas) aún cuando determinados funcionarios no lo hagan», uno debe leer «todo enunciado normativo es susceptible de valor de verdad sobre la base del hecho de que tiene un contenido proposicional (y esto es así, reitero, porque dicho enunciado puede ser afirmado)». En este sentido, el aspecto objetivo de la evocación de la pretensión así como de la necesidad es perfectamente compatible con cualquier deficiencia en las actitudes o intenciones de funcionarios particulares y con su fracaso en la evocación de la pretensión de corrección ${ }^{17}$.

15 Por «imparcialmente», entiendo aquí no un asunto de la psicología de un individuo particular, sino una cuestión de la psicología poseída por quien quiera que sea un participante en el sistema jurídico.

16 Desde luego la información sobre las intenciones del emisor es relevante para determinar el contenido de la proposición. Sin embargo, su importancia no debe ser exagerada: aun en el caso de expresiones extremadamente contenido-dependientes, tales como las expresiones indicativas, la mayoría de filósofos del lenguaje están dispuestos a reconocer que existen algunos contenidos de ejecución independiente que fijan la proposición en combinación con las circunstancias que rodean la emisión del enunciado relevante, el cual contiene la expresión indicativa. Es más, la pieza de ejecución independiente de la expresión indicativa es lo que guía la selección de aquellas piezas de información significantes para la individualización de la proposición, de entre aquellas piezas de información acerca de las intenciones de los emisores. Fundamental en esta vertiente es el trabajo de D. KAPLAN (especialmente KAPLAN, 1989).

17 Sobre este punto, vid. también la sección cuarta de este ensayo. 
Por otra parte, la necesidad de someter las proposiciones normativas a fundamentación implica que toda proposición será verdadera o falsa. Proposiciones normativas verdaderas constituirán obligaciones (normas) genuinas, mientras que aquéllas falsas no alcanzarán a ser distinguidas como normativas —ellas no serán consideradas como obligaciones (y aún más, ellas constituirían coacción sobre otros, si actuaran sobre ellos)—.

En este sentido, el APC establece la relación entre el derecho y la moral, al mostrar que la normatividad es una empresa cognitiva, sin tratar de demostrar que cada derecho, moral y demás deben ser considerados como normativos en el sentido de participar de una propiedad (no normativa) común (tal como la regla de un acto de habla) ${ }^{18}$. El punto de vista anterior presupondría que no existe tal cosa como el ámbito jurídico, que pueda ser constituido de manera antecedente a lo que cuenta como bases (normativamente sólidas). Sostener que la moral tiene relación con el concepto de derecho, no haría más que presentar una solución débil, referida a un filtro moral, que no alcanza a constituir una característica inherente al derecho.

\section{LA AFIRMACIÓN DE UNA PROPOSICIÓN NORMATIVA}

¿Cuáles serían las consecuencias de los diversos fracasos de la dimensión performativa de los enunciados normativos adecuadamente expresados al asumir el cognitivismo normativo puro? Éste será el tema de esta sección.

Dado el vocabulario de los actos de habla que más impregna la discusión sobre la pretensión de corrección, no sólo en ALEXY sino también en HABERMAS, es necesario aclarar las consecuencias que tiene una interpretación cognitivista de dicha pretensión, tal como ha sido propuesto en páginas anteriores. Aun cuando un profundo interés en el cognitivismo normativo ha dado a conocer la razón fundamental de la ética del discurso, es un hecho irrefutable que el particular vocabulario que ha sido empleado a través de los años por sus defensores, ha dado un fuerte apoyo a la impresión de que la posibilidad del cognitivismo depende de la fenomenología de la afirmación.

Afortunadamente, esa impresión es más débil de lo que parece, como he tratado de sostener en este escrito. No obstante, aún existe una justificada aporía en relación al impacto de la interpretación que propongo sobre la fenomenología de la pretensión de corrección: ¿En qué medida son modificadas las condiciones o las reglas de aserción de una proposición normativa, y con qué efecto?

Debido a la falta de espacio, tocaré sólo dos puntos: en primer lugar, voy a discutir las condiciones de éxito necesarias para afirmar un enunciado normativo. Segundo, ofreceré algunas nuevas respuestas con respecto a las consecuencias que resultan del fracaso de la afirmación adecuada de un enunciado normativo. En este caso, a pesar de que el fracaso de la afirmación adecuada de un enunciado normativo no puede tener un impacto radical en el contenido del enunciado, como algunas de las formulaciones de, entre otros, ALEXY nos conducen a pensar, existen aún algunos problemas relacionados con dicho fracaso que deben ser abordados.

18 Hay un dejo dworkiniano en esta empresa: la pretensión de que no es posible constituir primero la differentia specifica de cada dominio normativo y después otorgarles a ellos la fuerza práctica que es requerida. 
Una vez que la realización del acto de habla ha sido separado de su contenido proposicional, puede apreciarse que la pretensión de corrección no puede (al menos, no de manera exclusiva) relacionarse con el aspecto performativo de la aserción. En otras palabras, sería un error asumir que la regla de aserción de una proposición normativa corresponde a su pretensión de ser correcta. Porque si lo hiciéramos, despojaríamos al componente proposicional del acto de aserción de la importante virtud de dotar por sí mismo de corrección: esto es debido al importante hallazgo de que toda aserción normativa (o, mejor, la proposición que es afirmada) difiere los criterios de su firmeza o corrección al hecho normativo, al considerarlo como verdadero. Es más, como ya se puso de manifiesto anteriormente, si hiciéramos ello, estaríamos forzados, en un momento posterior en nuestro razonamiento, a retornar falazmente a la dimensión performativa, en orden a recuperar el criterio de corrección. Tal medida, sin embargo, llegaría al fatal resultado de la abolición de la distinción entre las actitudes del emisor y cualquier otro elemento mental independiente que debe fundamentar nuestros juicios. Perder la dimensión de la independencia mental equivaldría a descartar la posibilidad del cognitivismo (normativo), el cual es la idea singular más importante que la ética del discurso desde hace algún tiempo ha logrado poner en la agenda de nuestra forma de pensar y hablar en relación a la normatividad.

\subsection{Los requerimientos de la aserción}

Si se admite una explicación cognitivista de la pretensión de corrección, las condiciones de éxito necesarias para afirmar un enunciado normativo incluirán: en primer lugar, el requerimiento de que el contenido de la emisión esté dado por una proposición, cuando ésta resulta ser cierta (i. e. un hecho). Segundo, el requerimiento de que el emisor se encuentre en un estado mental que incorpore directamente el contenido de la proposición emitida ${ }^{19}$. Mientras la razón de la primera condición debería estar más bien clara por ahora, la razón de la segunda requiere ser explicada. El punto es que, cuando un emisor emite un enunciado normativo, él o ella debe encontrarse en un estado mental que es determinado por la proposición la cual individualiza su emisión, sin la mediación de la absorción mental del emisor (esto es, su «impresión», o «idea») de la situación particular.

En contraste con la creencia de que, lo que es requerido es un estado mental «introvertido» —aunque aspire a reflejar con precisión el mundo-, lo que es necesario es un estado de la mente que sea «externalista», en el sentido de que su contenido no sea determinado por las características mentales del emisor, sino por lo que hace una proposición verdadera (esto es, un hecho). El caso más importante de tal estado de la mente es el conocimiento. Al configurar esta condición, la segunda regla de aserción asume la forma de «la regla de conocimiento». Ésta dice:

19 AleXY, en su ensayo «Law and Correctness» (ALEXY, 1998), parece agregar un tercer requerimiento, el cual está basado en las relaciones entre emisores: de acuerdo con él, afirmar un enunciado también implica que el emisor presenta a su audiencia una garantía de posible justificación, la cual además está conectada con una expectativa de que todo destinatario aceptará la proposición jurídica como verdadera. Según mi opinión, esto no determina un componente separado del acto de aserción, si es asumido que este último descansa sobre la regla según la cual se requiere que el agente se encuentre en un estado mental, el cual se adhiere al contenido proposicional de la emisión (vid. también la discusión en HEIDEMANN, 2005, 131-133). 
(La regla de conocimiento) Se debe: afirmar $p$ sólo si se conoce $p^{20}$.

Con la regla de conocimiento, se configura de manera consistente la primacía de lo proposicional sobre la dimensión performativa de la aserción. Debido a que, con base en la regla de conocimiento, la ejecución de una aserción es correcta sólo si ella está fundada sobre un hecho normativo.

Por consiguiente, conforme a la interpretación que propongo, la pretensión de corrección comprende dos requerimientos o reglas: la regla del contenido proposicional y la regla de conocimiento. Mientras que la primera regla raras veces puede ser violada (e. g. cuando existe un claro desvío del discurso estructurado, o una consecuente privación del discurso) la segunda regla, la regla de conocimiento, puede ser violada en distintos momentos, en particular cuando el emisor adopta alguna otra actitud o cuando explícitamente se propone contradecirla. Sin embargo, cualquier falta de cumplimiento de la regla de conocimiento, aun cuando destruya el carácter del acto como acto de aserción, no puede despojarlo de su contenido proposicional y, por consiguiente, de tener que rendir cuentas ante aquellos hechos normativos que lo hacen verdadero o falso. La razón es simple: al basarse en la regla de conocimiento, la parte performativa se subordina a la dimensión proposicional. Así que, cuando la primera es socavada, sus efectos no se extienden a esta última, que continúa ejerciendo su efecto normativo: aun los dictadores, los abogados desilusionados o los calculadores tecnócratas siguen siendo responsables en relación a lo que es correcto o incorrecto, por lo que se puede decir que ellos están sujetos tanto a la evaluación normativa como a los hechos y dichos de cualquier persona que pretenda tener en cuenta el pleno de las consecuencias normativas de los pronunciamientos de ellos.

\subsection{Los fracasos al afirmar adecuadamente}

Pasaré ahora a examinar en mayor detalle algunas de las consecuencias de la falta de cumplimiento del segundo componente de la pretensión de corrección, esto es, la regla de conocimiento. ¿Qué ocurre cuando se niega o deja de actuar de acuerdo con la regla: «Afirma sólo aquello que conoces»?

i) Si la proposición normativa que se plantea es cierta (esto es, corresponde a un hecho normativo, o a una norma) entonces el daño es pequeño. Éste es el caso de un juez que dice: «Tú tienes que $\mathrm{F}$, aun cuando yo pienso que hacer $\mathrm{F}$ es inmoral». En este caso, fuera del fracaso personal del juez, no se ha hecho realmente daño a los destinatarios de la ley ${ }^{21}$. Para ilustrar el punto mejor: piénsese en un juez moralista que pronuncia con resentimiento: «Las parejas homosexuales tienen derecho a adoptar niños, aunque esto es inmoral». Este fracaso de la afirmación apropiada de un enunciado normativo no hace su contenido falso de ninguna manera. De hecho las partes del caso estarían probablemente del todo satisfechas con la sentencia.

${ }^{20}$ En relación a la descripción cognitiva de la aserción y, de manera más amplia, a la poderosa reconstrucción del conocimiento como estado de la mente, vid. el trabajo fundamental de T. WiLliamson (WiLLiamson, 2000, caps. 1, 11).

${ }^{21}$ Es una cuestión diferente si se está exigiendo los daños causados por el hecho de que los jueces yerren al reconocer lo que es correcto. Lo que estoy examinando en el caso anterior es el impacto de la emisión judicial que es pronunciada. 
ii) Si la proposición normativa que es afirmada es falsa (el hecho normativo/ norma no existe), entonces, en la medida en que tiene un impacto en la vida de las personas, resultaría ser un caso de coacción pura. Así por ejemplo el juez que dice: «Tú debes seguir el Führenprinzip, aun cuando esto es inmoral». Esta emisión no da lugar a ninguna obligación (jurídica), precisamente porque la proposición que individualiza su contenido es falsa. Es interesante comparar este caso con respecto a sus resultados con el caso en el que R. FREISLER afirma adecuadamente el Fübrerprinzip. De nuevo aquí lo que se obtiene es un caso de pura e ilegítima coacción.

iii) Por último, quisiera examinar brevemente el fracaso de la dimensión performativa de la aserción en sí misma considerada, lo que es independiente de si la proposición que es afirmada es verdadera o falsa. ¿Existe un caso de un error más general, que aquél en el cual alguien deja de afirmar una proposición normativa adecuadamente? Ilustraré el punto por medio de una referencia a otra influyente teoría jurídica que acoge una versión de la pretensión de corrección entendida como marco conceptual del derecho: tengo en mente el modelo de autoridad de J. RAZ, el cual pone a la pretensión de corrección al servicio de una posición positivista fuerte. De acuerdo con RAZ, todo sistema jurídico tiene la pretensión de poseer una autoridad legítima como una necesidad conceptual. Por consiguiente, plantean dicha pretensión no sólo aquellos sistemas, que son de hecho legítimos, sino también aquéllos ilegítimos, que sin embargo comparten algunas características no normativas comunes con los primeros. En palabras de RAZ:

Un sistema jurídico puede carecer de autoridad legítima. Si éste carece de atributos morales necesarios para dotarlo de una autoridad legítima entonces no tiene ninguna. Sin embargo, él debe poseer todas las otras características de la autoridad, o de lo contrario sería extraño decir que él tiene una pretensión de autoridad. Para pretender tener autoridad, él debe ser capaz de tenerla, debe ser un sistema de la clase de aquéllos que son capaces en principio de poseer el requisito de las propiedades morales de la autoridad (RAZ, 1996, 215).

Él especifica las características que hacen un sistema de la clase de aquellos que es capaz en principio de poseer autoridad legítima, como sigue:

Las dos características son las siguientes. En primer lugar, una orden puede resultar autoritativamente obligatoria sólo si representa el punto de vista de una persona acerca de cómo deben comportarse sus gobernados, o al menos si es presentada de esta manera. En segundo lugar, debe ser posible identificar la orden como emanada por la supuesta autoridad, independientemente de otras razones o consideraciones que la orden se proponga resolver (RAZ, 1996, 218).

Con esta estrategia RAZ aísla las órdenes jurídicas de los fundamentos que hacen su componente proposicional verdadero o falso. No obstante, esta jugada tiene además la consecuencia de despojar a las órdenes también de su dimensión performativa: si la pretensión de poseer una autoridad legítima es reducida a la existencia del mandato de alguien, más una confianza directa en el mandato (esto es, sin referencia alguna a una razón de fondo), entonces ya no tiene relevancia la pretensión de poseer una autoridad legítima, si el agente afirma sólo lo que él conoce ser el caso. Tan pronto como, no obstante, se ha despojado a la pretensión de poseer autoridad legítima de un aspecto proposicional y uno performativo por igual, dicha pretensión comienza a disolverse en el aire. 
No obstante, sin dicha pretensión, lo que el mandato de una autoridad cualquiera representará será coacción pura. Dado que pronunciar un enunciado normativo es en sí mismo una acción, esto hace que se encuentre sujeto a las mismas normas que gobiernan todo tipo de acción. De esto se sigue que toda declaración de una norma que no se guíe por el conocimiento de que la orden es una norma (correcta), configurará probablemente una imposición de fuerza sobre otros ${ }^{22}$.

En conclusión ${ }^{23}$, una descripción cognitivista pura de la pretensión de corrección sostendría que, debido a la dimensión proposicional de la pretensión, todos los enunciados normativos están sujetos a una evaluación normativa sobre la base de los hechos normativos que hacen verdaderas o falsas las proposiciones relevantes. Con respecto al aspecto performativo de la pretensión, que es la regla de conocimiento, los siguientes casos deben ser distinguidos: si el emisor no sigue la regla, entonces el emisor no obstante eleva la pretensión de corrección a través de la dimensión proposicional de la pretensión. Sin embargo, el fracaso en la evocación de la pretensión puede constituir un yerro moral en sí mismo, para el caso de la parte performativa de la pretensión, esto indica que los actos de habla normativos son en sí mismos acciones, y en cuanto a tales, pueden ser considerados verdaderos o falsos, dependiendo de si ellos están de acuerdo o violan las normas existentes (como hechos normativos).

(Traducción de Gonzalo Villa Rosas)

\section{REFERENCIAS}

AleXy, R., 1998: «Law and Correctness», Current Legal Problems, 51: 205-221 (versión en español: ALEXY, R., 2010: «Derecho y corrección», La institucionalización de la justicia, Comares, 28-47).

- 1999: «My Philosphy of Law. The Institutionalisation of Reason», The Law: in Philosophical Perspectives, Luc Wintgens, 23-46. Dordrecht: Kluwer (versión en español: ALEXY, R., 2000: «La institucionalización de la razón», Persona y Derecho, Universidad de Navarra, 43: 217-249).

- 2002a. The Argument from Injustice, Oxford: Clarendon (versión en español: Alexy, R., 1994: El concepto y la validez del derecho, Barcelona: Gedisa).

- 2002b. A Theory of Constitutional Rights, Oxford University Press (versión en español: Alexy, R., 2002: «Epílogo a la teoría de los derechos fundamentales», Revista Española de

${ }^{22} \mathrm{El}$ contraste con el modelo de autoridad es instructivo, en la medida en que puede servir como una advertencia: los diversos intentos por evitar el aspecto propositivo de la pretensión de corrección lo más probable es que terminen también en una situación en la cual, la dimensión performativa es indeterminada (así por ejemplo, si se reduce al visto bueno de alguien). No obstante, en ausencia de las dos dimensiones, el derecho se convierte en un sistema de coerción pura.

${ }^{23}$ Hay además una pregunta relacionada con el impacto de la dimensión performativa de la pretensión sobre el estatus de las proposiciones normativas. Ésta concierne a la cuestión de si la ejecución correcta de la aserción puede afectar los fundamentos de la proposición que es afirmada. Recientemente S. DARWALL (DARWALL, 2007a) ha afirmado que las razones morales, en virtud de su carácter recíproco o relacional, están fundadas en la perspectiva segunda personal, que se plantea por las pretensiones que son presentadas por personas de manera recíproca. No puedo discutir esta perspectiva aquí en detalle. Mi propio punto de vista al respecto, es que el carácter recíproco de las normas morales, que es sin duda el caso, puede ser fundado de manera satisfactoria en la perspectiva primera personal que es generada por la capacidad reflexiva de las personas. Para una crítica a DARWALL, que se basa en una premisa similar, vid. Ch. KORSGAARD (KORSGAARD, 2007) y la respuesta de DARWALL (DARWALL, 2007b). 
Derecho Constitucional, Madrid: Centro de Estudios Políticos y Constitucionales, 22: 13-64. 2007: reimp. en Teoría de los derechos fundamentales, 2. ${ }^{a}$ ed., Madrid: Centro de Estudios Políticos y Constitucionales, 511-562).

- 2007: «13 Replies», Law, Rights and Discourse, G. Pavlakos (ed.), Oxford and Portland: Hart, 333-336.

ApEL, K.-O., 1998: Auseinandersetzungen in Erbrobung des Transzendental-prgmatischen Ansatzes, Frankfurt am Main: Suhrkamp.

Bulyguin, E., 1993: «Alexy und das Richtigskeitsargument», Rechtsnorm und Rechtswirklichkeit, Aarnio, A.; Paulson, S. L.; Weinberger, O.; von Wright, G. H., y Wyduckel, D. (eds.), Berlin: Duncker \& Humblot, 19-24.

— 2000. «Alexy's Thesis of the Necessary Connection between Law and Morality», Ratio Juris, 13: 133-137.

Darwall, S., 2007a: The Second-Person Standpoint, Cambridge Mass: Harvard University Press.

— 2007b: «Reply to Korsgaard, Wallace and Watson», Ethics, 118: 52-69.

FitZPATRICK, W., 2009: «Recent Work on Ethical Realism», Analysis, 69: 746-760.

Habermas, J., 2001: «Discourse Ethics: Notes on a Programme of Philosophical Justification», en J. HABERMAS, Moral Conscioussness and Communicative Action, 43-115, Cambridge Massachusetts: MIT Press (versión en español: HABERMAS, J., 1987: «La ética del discurso: notas sobre un programa de fundamentación», Conciencia moral y acción comunicativa, Madrid: Península, 59-134).

HeIDEMANN, C., 2005: «Law's Claim to Correctness», Jurisprudence or Legal Science?, CoyLE, S., y Pavlakos, G. (eds.), Oxford and Portland: Hart, 127-146.

Kaplan, D., 1989: Demonstratives. An Essay on the Semantics, Logic, Metaphysics, and Epistemology of Demonstratives and Other Indexicals, reimpreso en Themes from Kaplan, ALMOG, J. et al. (eds.), New York: Oxford University Press, 481-563.

KorsGaARD, Ch., 2007: «Autonomy and the Second Person Within: A Commentary on Stephen Darwall's the Second Person Standpoint», Ethics, 118: 8-23.

PARFIT, D., 2006: «Normative Reasons», Oxford Studies in Metaethics, vol. 1, Russ Shafer-Landau, Oxford: Clarendon, 325-380.

Pavlakos, G., 2009: «Practice, Reasons, and the Agent's Point of View», Ratio Juris, 22: 74-94.

- 2011: «Law, Normativity and the Model of Norms», New Essays on the Normativity of Law, Bertea, S., y Pavlakos, G. (eds.), Ch. 11. Oxford and Portland: Hart Publishing.

RAz, J., 1996: «Authority, Law and Morality», en RAz, J., Ethics in the Public Domain, edición revisada, Oxford: Clarendon Press, 210-237 (versión en español: RAZ, J., 2001: «Autoridad, derecho y moral», La ética en el ámbito público, Barcelona: Gedisa, 227-243).

Recanati, F., 2004: Literal Meaning, Cambridge: Cambridge University Press.

Schroeder, M., 2008: Being For: Evaluating the Semantic Program of Expressivism, Oxford: Oxford University Press.

Williamson, T., 2000: Knowledge and its Limits, Oxford: Oxford University Press. 